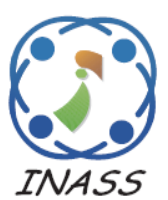

\title{
Hybrid Model Combined Fuzzy Multi-Objective Decision Making with Feed Forward Neural Network (F-MODM-FFNN) For Very Short-Term Load Forecasting Based on Weather Data
}

\author{
Eppy Yundra ${ }^{1}$ \\ Unit Three Kartini ${ }^{1 *}$ \\ Laili Ika Wardani ${ }^{1}$ \\ Dwi Ardianto 1 \\ ${ }^{I}$ Department of Electrical Engineering, Universitas Negeri Surabaya, Surabaya, Indonesia \\ * Corresponding author’s Email: unitthree@unesa.ac.id
}

\begin{abstract}
This research paper proposes a new hybrid methodology for very short-term load forecasting of hourly or designed to predict load for 1 hour ahead. The proposed hybrid methodology is based on weather data especially for optimizing the operation of power generating electricity from thermal generation. This hybrid modelling is a combination of the Fuzzy-Multi-Objective Decision-Making and feed-forward-Neural Network method. The novelty of this hybrid model is taking into account the weather and meteorology data. The first hybrid model implements Fuzzy-Multi Objective Decision Making as an input data pre-processing technique before the feed-forward Neural Network model. The error statistical indicators of the Fuzzy-Multi Objective Decision Making-Feed Forward Neural Network) model for mean square error is the mean value error 0.001 and root mean square error is mean value error 0.2. Note that the highest root means square error was $4.43 \mathrm{MW}$ and the mean square error was $837.8 \mathrm{MW}$ during the sixteen periods.
\end{abstract}

Keywords: Load, Forecasting, Thermal generation, Fuzzy-multi objective decision making-feed forward neural network, Hybrid, Modelling, Weather.

\section{Introduction}

Nowadays, short term load forecasting is an essential task to perform, particularly related to the thermal generation energy with the interconnection system as a source of power electrical. Since thermal generation using gas fuel, coal fuel, and gas oil fuel is an electrical power generation system is categorized as primary energy sources, the shortterm load forecasting is paramount to regulate electricity load in power networks. It also functions to maximum power delivery, unit commitment, economic dispatch, minimization loss transmission, and minimize the operating cost of the power system. This proposed the research presents a section of research namely short-term load forecasting using Fuzzy Multi-Objective Decision Making - Neural Network based on weather data for 60 minutes ahead. In this research, a novel methodology that combines Fuzzy Multi-Objective Decision-Making modelling and Artificial Neural Network modelling has been developed for short term load forecasting. Fuzzy Multi-Objective Decision Making Feed Forward Neural Network modelling is used to short term load forecasting at the thermal generation by determining the rule, membership function, and fuzzy set-based multiobjective decision making and then do the testing and training data using Artificial Neural Network modelling.

The performance of thermal generation center systems is heavily influenced by weather conditions namely load, fuel, temperature, and humidity. With short term load forecasting based on weather data, it is expected that operation control power systems can be improved, to balance energy electrical power generation and load. The result of electrical energy production by steam generation, gas generation and combined cycles generation system depending on weather, time, and load. Previous research has presented a variety of mathematical methods for load forecasting about meteorological data variables. 
The performance of thermal generation systems is heavily influenced by some weather conditions data, consisting of fuel, temperature, lead generation, and humidity. The relation is clear: electrical energy generated by the thermal generation depends on the amount of the fuel used by thermal unit generation. Output power electrical energy depending on the temperature, humidity, and fuel, because the more fuel used, the higher the heat rate value and the greater the output power from thermal generation. Previous research has presented and explains a variety of mathematical methods for load forecasting about weather variables. The result of the electrical energy production steam and gas generation system depending on weather and meteorology, time, load customers and geographic location. Previous research has presented a variety of mathematical methods for load forecasting about meteorological variables. D. Liu, L. Zeng, C. Li, K. Ma Y. Chen and Y. Cao [1] presented a load forecasting method based on local weather information for a distributed short term, which includes neural network, autoregressive integrated moving average model, autoregressive and moving average and gray model. Y. Wang, Q. Chen, M. Sun, C. Kang, and Q. Xia [2] presented a novel ensemble method for load forecasting, where the sub-profiles are first clustered in different groups and forecasting is conducted on the grouped load profiles individually. T. Vincent, A. Pichavant, Y.Goude A. Antoniadis and J. M Poggi [3] presented about prediction a semi-parametric based on additive models using consider an application to the customer load demand at an aggregate level. Short term load forecasting and operation strategy probabilistic design using the combination model, has been described in [4-6]. Load forecasting design using Forecasted Loads by M. Liu, Y. Shi and F. Fang [4]. Load Forecasting and normalization by $\mathrm{T}$. Hong, J. Wilson, J. Xie and Long [5]. Quantile Regression for probabilistic load forecasting by B. Liu, J. Nowotarski, T. Hong, and R. Weron [6]. B.Li, J. Zhang, Y. He and Y. Wang [7] presented shortterm load forecasting using wavelet decomposition with a neural network model. K. Weicong, D. Z. Yang, D. J. Hill, L. Fengji, and Y. Xu [8] have presented load forecasting for short term residential based on resident behavior learning. Load prediction is the main problem in the reliability electrical power system, where the data load in the form of the time series can also be analyzed using temperature scenario generation as described in reference [9]. J. Xie and T. Hong [9] have presented about the various approaches to generating probabilistic load forecasts, feeding simulated weather scenarios to a point load prediction model is being commonly accepted by the industry for its simplicity and interpretability. The efforts involved in improving the system level intraday load prediction by applying clustering to identify groups of customers with similar load consumption patterns from smart meters before performing load forecasting as described in reference [10]. L. Franklin, L. W. Jen, H. Heng, Y. D. Wang, and L. S. Robert [10] worked on the efforts involved in utilizing the data to improve the load prediction accuracy at the system level. Short term load forecasting is not only using the classical method, where the fisher information based meteorological factors are the main input variables as described in reference [11]. S. Cai, L. Liu, H. Sun, and J. Yan [11] have presented the weather information is a main important factor in short term load forecasting, and this paper is to develop a novel methodology based on fisher information for meteorological factors and variables introduction and variable selection in short term load forecasting. B. Wang, Y. Li and J. Watada [12] have presented about supply reliability and generation cost analysis due to load forecast uncertainty in unit commitment problems. U. T. Kartini, D. Ardianto, and W. Laili [13] worked explain about modeling and simulation Very ShortTerm Load Forecasting Based on Meteorological With Modelling k-Nearest Neighbour-Feed Forward Neural Network for hydrothermal generation in Indonesia. Optimization and analysis data design using the combination Multi-Objective Decision Making model, has been described in [14-20]. Optimization solar/wind penetration at energy renewable using Multi-Objective by F. Mohamad, J. The, and H. Abunima [14]. Analysis data hierarchy Process based on Multi-Criteria Decision Making Method by D. J. Yi Tey, Y. F. Gan, G. Selvachandran, S. G. Quek, F. Smarandache, L. H Son, M. A. Basset and H. V Long [15]. MultiObjective Finite for predictive control by S. M. A. Pahnehkolaei, H. Vahedi, A. Alfi, and K. AlHaddad [16]. A Centroid Decision Making Approach For Solving A Multi-Objective Optimal Power Flow by E. Barocio, J. Regalado, E. Cuevas, F. Uribe, P. Zuniga, and P. J. R Torres [17]. Short Term Voltage Security of Power Systems Using Accelerated Multi-Objective Reinforcement Learning by Z. Deng, Z. Lu, Z. Guo, W. Yao, W. Zhao, B. Zhao, and C. Hong [18] and A Stochastic Multi-Objective Framework For Optimal Scheduling of Energy Storage Systems by H. Farzin, M. F. Firuzabad, and M. M Aghtaie [19]. Multiobjective Expansion Planning Approach: Distant Wind Farms by M. S. Javadi, M. Saniei, H. R. 
Mashhadi, and G. G Alcaraz [20]. H. Khazraj, B. Y Khanghah, P. Ghimire, F. Martin, M. Ghomi, F. F. Silva, and C. L. Bak [21] have presented about the management optimal operational scheduling and reconfiguration based on weather condition using a hybrid optimization algorithm based on genetic algorithm set. Multi-Objective Decision-Making model for Planning problem has been described in [22, 23]. H. Mavalizadeh, A. Ahmadi, and A. Heidarr [22] have presented probabilistic multiobjective generation and transmission expansion planning problem using normal boundary intersection. S. M. Mazhari, H. Monsef, and R. Romero [23] have presented planning incorporating customer choices on reliability using multi-objective generation and transmission expansion planning problem using normal boundary intersection. S. Motepe, A. N. Hasan and R Stopforth [24] worked to explain about load forecasting process for a power distribution network using the hybrid model. And application optimization reactive power dispatch of power systems based on multi-objective decision making has been described by $\mathrm{M}$. Zhang and Y. Li [25]. Optimization power scheduling, modeling, planning, and forecasting based on the mathematic model, has been described in [26-31]. Optimal power scheduling of smart residential buildings by A. Ouammi [26]. Modeling and forecasting short term power load using deep learning by T. Ouyang, Y. Ho, H. Li, Z. Sun, and S. Back [27], Optimization based on Fuzzy by Y. Shen and G. Ge [28], Multi-objective Synergistic Planning of EV Fast-Charging Stations In The Distribution System Coupled by A. Shukla, K. Verma, and R. Kumar [29]. Scheduling thermal generation day ahead using evolutionary multiobjective by A. Trivedi, D. Srinivisan, D. Sharma, and C. Singh [30]. And coordinated multi-objective decision making for multiple Trade-Offs has been described as Z. Zhao, J. Yang, W. Yang, H. Luo, and Y. Peng [31].

This paper aims and presents focuses a part of the research in progress that seeks to estimate load generation thermal unit production one hour ahead. This part concerns how to forecast the hourly load generation thermal unit for a given site based on meteorology data. In this research, a novel methodology that combines Fuzzy Multi-Objective Decision Making (F-MODM) modelling and the Feed-Forward neural networks (FFNN) modelling techniques have been developed. A Fuzzy MultiObjective Decision Making Feed Forward Neural Networks is used to model the load thermal generation using a daily forecast provided by another unit thermal generation.
Fuzzy Multi-Objective Decision Making Feed Forward Neural Network (F-MODM-FFNN) modelling is used to short term load forecast at the thermal generation by decision-making rule fuzzy logic based on meteorology data and then do the testing and training data at feed-forward neural network model. This paper proposes a time series forecasting model that integrates Fuzzy MultiObjective Decision Making and Feed Forward Neural Network model algorithms. The Fuzzy Multi-Objective Decision-Making model is used to search for the optimal weights data and the Feed Forward Neural Networks is used to search for the predict load generation thermal unit. The model is tested and compared with k-nearest neighbor Neural Network and Artificial Neural Network, where the conventional model is used to search for similar time series, while the advantages our model the load forecasting based on meteorology data can get more accurate forecasting results.

The remainder of the article is organized as follows: Section 2 describes the modelling and data from thermal unit generation, section 3 describes the combination methodology used, i.e. Fuzzy MultiObjective Decision Making Feed Forward Neural Network method and Feedforward neural network model, while Section 4 presents study cases of the modelling in very short-term load forecasting and its measured error. And finally, Section 5 presents some concluding remarks.

\subsection{Notation}

The notation used throughout the paper is stated below.

Indexes:
$\mu_{\bar{N}}(x)$
is the continuous mapping from
$\mathrm{R}$ to the interval of $[0,1]$
$\mu_{\bar{N}}(x)$
are the convex fuzzy subset and the normalization of a fuzzy subset which means that there exists a number $x_{0}$ makes $\mu_{\overline{\mathrm{N}}}(\mathrm{x})$ is amount 1
$k \quad$ are the alternatives
$Y \quad$ is the modal value
$Z$ and $x$ the lower and upper bounds of the fuzzy number

\section{Constans:}

$$
\begin{array}{ll}
n_{i}(x) \quad \begin{array}{l}
\text { indicates the satisfaction of } \\
\text { alternative } x \text { to objective } n_{i} \\
\text { given a set of } d \text { objectives or } \\
\text { criteria }
\end{array} \\
X_{i}, Y_{i} \quad \begin{array}{l}
\text { The i-th matrix corresponding } \\
\text { scenarios composed }
\end{array}
\end{array}
$$




\subsection{System description and data analysis data collection}

In this research, measured the load hourly data and weather data are used to forecast the load generation for the next hours [12]. The load generation measurements were performed continuously every 30 minutes in sixteen hours. The dataset for short term load forecasting contains thirty four hours of data (from 00.30 a.m. to 04.00 p.m) on 20-March 2018 at twelve the thermal generation using gas fuel and between one thermal generation with another thermal generation in interconnection system: combined cycle Gas and Steam power plant GT 11 (112,45 MW), combined cycle Gas and Steam power plant GT $12(112,45$ MW), combined cycle Gas and Steam power plant GT 13 (112,45 MW), combined cycle Gas and Steam power plant ST 10 (188,91 MW), combined cycle Gas and Steam power plant GT $21(112,45$ MW), combined cycle Gas and Steam power plant GT 22 (112,45 MW), combined cycle Gas and Steam power plant GT 23 (112,45 MW), combined cycle Gas and Steam power plant ST $20(188,91$ MW), combined cycle Gas and Steam power plant GT 31 (112,45 MW), combined cycle Gas and Steam power plant GT 32 (112,45 MW), combined cycle Gas and Steam power plant GT $33(112,45$ MW), combined cycle Gas and Steam power plant ST 30 (188,91 MW). Fig. 1 illustrates daily load data values as measured at thermal generation. These monitored data have been used to validate the methodology using Fuzzy-Multi Objective Decision Making -Feed Forward Neural Network as the proposed model for very short term load forecasting.

The area usage-based Fuzzy-Multi Objective Decision Making -Feed Forward Neural Network modelling load forecasting computer simulation has been proposed and based on weather and meteorology information to simulate future load growth based on different anticipated scenarios. The increasingly popular, affordable, and accurate weather data provide an excellent database platform for Fuzzy-Multi Objective Decision Making -Feed Forward Neural Network hybrid modelling load forecasting techniques. Thus Fuzzy-Multi Objective Decision Making -Feed Forward Neural Network modelling for load forecasting technology becomes even more attractive than before both from an economic point of view and superior load forecasting based on weather and meteorology data accuracy. There are a few stages for Fuzzy-Multi Objective Decision Making -Feed Forward Neural Network load forecasting as shown in Fig. 2, where

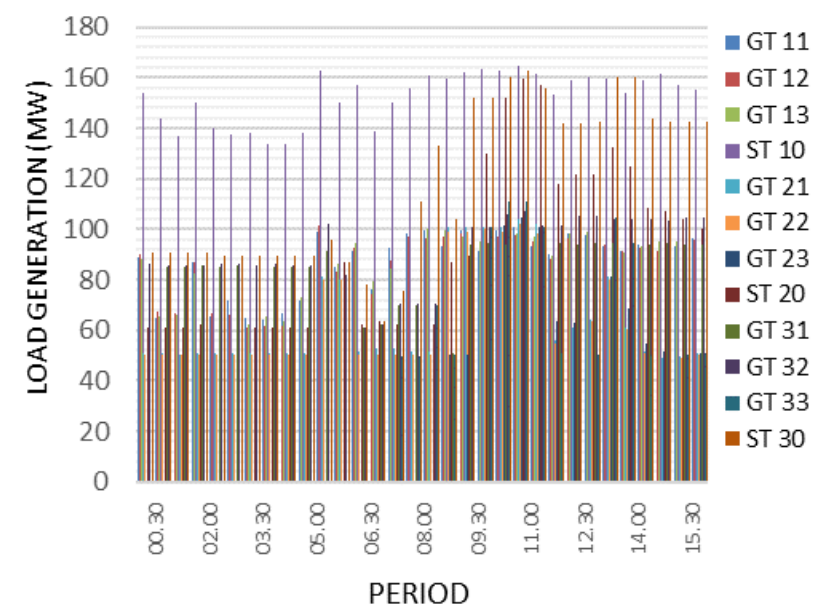

Figure. 1 Measured load data at thermal generation from 00.30 a.m. to 04.00 p.m.

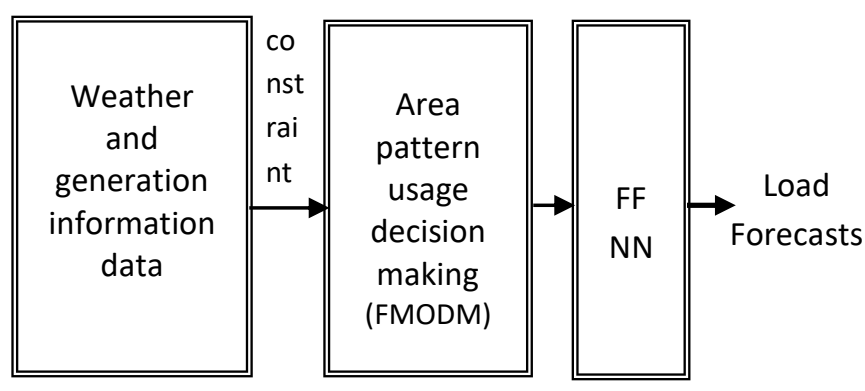

Figure. 2 Load forecasts for predicting the generated thermal power

load forecasts are used to predict the thermal power plant that a given combined cycle Gas and Steam power plant which fused in the interconnection system can generate utilizing its mathematical modelling. Fuzzy-Multi Objective Decision Making -Feed Forward Neural Network information is used to predict the load. The area usage is mapped to a load growth pattern recognition. The area usage and load growth are then calibrated based on different rule constraints, such as system load growth, fuel available, fuel cost, future economic growth of the area, humidity, temperature, etc.

\section{The methodology}

In this research, we propose a novel forecasting model that can improve the temperature and humidity prediction by sharing the strength of several meteorology services and this section explains the basic idea of the developed combination methodology for load forecasting, namely Fuzzy-Multi Objective Decision Making Feed Forward Neural Network hybrid model. The first purpose of this research is the improvement of load forecasting results using Fuzzy Multi-Objective Decision Making (F-MODM) model which 


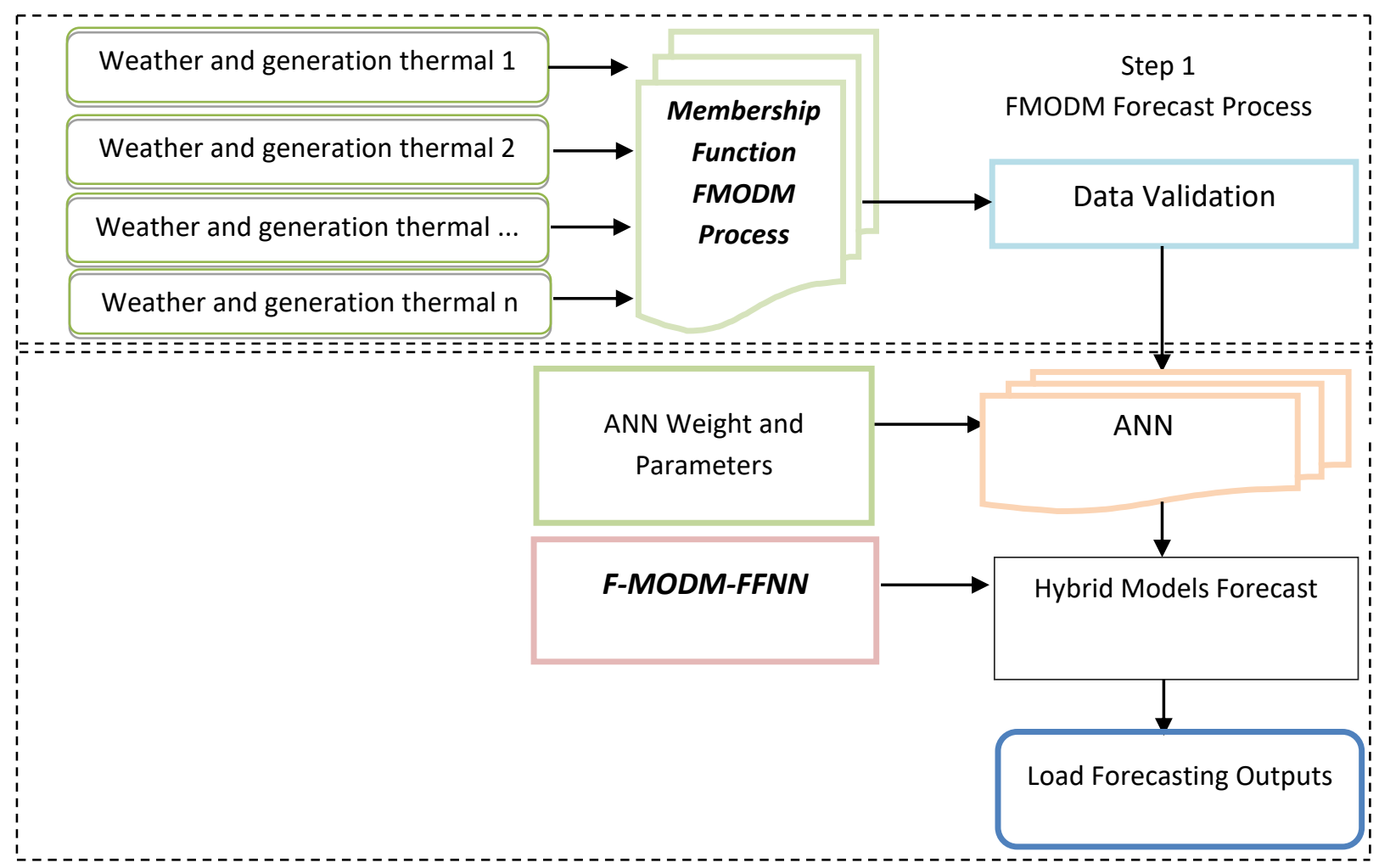

Figure. 3 Structure of comprehensive hybrid combination Fuzzy-Multi Objective Decision Making-Feed Forward Artificial Neural Network (F-MODM-FFNN) load forecasting model

combined with a model of feedforward artificial neural network (FFNN) methods, and the process is then used to forecast load output of a thermal generation one hour ahead based on weather data. Simulation of the Fuzzy-Multi Objective Decision Making -Feed Forward Neural Network can be programmed in a few minutes after the recording of the initial measurements. For the load forecasting, the Fuzzy-Multi Objective Decision Making -Feed Forward Neural Network hybrid combination method employs weather data i.e. power, temperature, fuel, and humidity.

\subsection{System structure}

The block diagram of the proposed load forecasting using a hybrid combination modelling system is shown in Fig. 3. Especially, the hybrid combination models system works under a two-step operation mode. In the first step, the raw data meteorology from different weather services and the raw data generation power system are gathered together which is then used as input data of the Fuzzy Multi-Objective Decision Making (FMODM) Feed Forward Artificial Neural Network (FFNN) models forecasting, and in the second step, hybrid combination models to generate an improved load forecast, in which each Fuzzy-Multi Objective
Decision Making -Feed Forward Neural Network has been created and integrated based on weather and meteorology data to produce the load forecasting. Process for the comprehensive hybrid combination Fuzzy Multiobjective Decision Making -Feed Forward Artificial Neural Network load forecasting modelling, there are two-steps, i.e: First Step: Concept of the Fuzzy Multi-Objective Decision Making (F-MODM). This section constructs an analytical model for measuring the prediction possibility of success of knowledge load forecasting using the Fuzzy Multi-Objective Decision-Making approach. We can see Fig. 4. The procedures for load forecasting implementation based on weather and meteorology data. Fuzzy Multi-objective decision making comprises a finite set of alternatives, among which the decisionmakers have to select, evaluate or rank according to the weights of a finite set of criteria (attributes). The purpose of this section is to establish a hierarchical structure for tackling the evaluation problem of load forecasting. This section comprises four subsections: investigating the parameters affecting knowledge load forecasting, building the hierarchical structure of evaluation criteria, determining the evaluation criteria weights criteria weights and determining the prediction possibility value load forecasting the results. 
Second Step: Concept of the Fuzzy Multi-Objective Decision Making (F-MODM) - FFNN

\section{Fuzzy Sets and Fuzzification}

A fuzzy set is a set containing elements that have varying degrees of membership in the set [Moyuen]. And the fuzzy numbers are the fuzzy subset of real numbers representing the expansion of the confidence. The fuzzy number $\tilde{X}$ be a fuzzy number set, of which the membership function is $\mu_{\tilde{B}}(x): R \rightarrow[0,1]$, and it comprises the following features:

1) $\mu_{\widetilde{N}}(x)$ is the continuous mapping from $\mathrm{R}$ to the interval of $[0,1]$

2) $\mu_{\widetilde{N}}(x)$ is the convex fuzzy subset

$\mu_{\widetilde{N}}(x)$ is the normalization of a fuzzy subset which means that there exists a number $x_{0}$ makes $\mu_{\widetilde{N}}(x)$ is amount 1

$$
\begin{aligned}
& \tilde{B}=\left[\begin{array}{ccccc}
1 & \tilde{b}_{12} & \tilde{b}_{13} & \cdots & \tilde{b}_{1 m} \\
\tilde{b}_{21} & 1 & \tilde{b}_{23} & \cdots & \tilde{b}_{2 m} \\
\tilde{b}_{31} & \tilde{b}_{32} & 1 & \cdots & \tilde{b}_{3 m} \\
\cdots & \cdots & \cdots & \ddots & \cdots \\
\tilde{b}_{m 1} & \tilde{b}_{m 2} & \tilde{b}_{m 3} & \cdots & 1
\end{array}\right] \\
& =\left[\begin{array}{ccccc}
1 & \tilde{b}_{12} & \tilde{b}_{13} & \cdots & \tilde{b}_{1 m} \\
1 / \tilde{b}_{12} & 1 & \cdots & \cdots & \tilde{b}_{2 m} \\
1 / \tilde{b}_{13} & 1 / \tilde{b}_{23} & 1 & \cdots & \tilde{b}_{3 m} \\
\cdots & \cdots & \cdots & \ddots & \cdots \\
1 / \tilde{b}_{1 m} & 1 / \tilde{b}_{1 m} & 1 / \tilde{b}_{1 m} & \cdots & 1
\end{array}\right]
\end{aligned}
$$

So, if those numbers fulfill the aforementioned mathematics method requirements then they are called fuzzy numbers has been explained by $\mathrm{H}$. Mavalizadeh, A. Ahmadi, and A. Heidarr [22]. The characteristics and membership function of the triangular fuzzy number (TFN) should possess the following basic features. The fuzzy number $A$ on $\mathfrak{R}$ to be the triangular fuzzy number if its membership function $\mu_{\tilde{A}}(x): R \rightarrow[0,1]$ is equal to

$$
\mu_{\tilde{A}}(x)=\left\{\begin{array}{cc}
(x-Z) /(Y-Z) & , Z \leq x \leq Y \\
(x-x) /(x-Y) & , Y \leq x \leq x \\
0, & \text { otherwise }
\end{array}\right.
$$

Where $\mathrm{Z}$ and $\mathrm{x}$ stand for the lower and upper bounds of the fuzzy number $\tilde{A}$ and $\mathrm{Y}$ is the modal value. The triangular fuzzy numbers (TFNs) can be denoted by, $\tilde{A}=(Z, Y, x)$ and the following are the operational laws of two the triangular fuzzy numbers $\widetilde{A_{1}}=\left(Z_{1}, Y_{1}, x_{1}\right)$ and $\widetilde{A_{2}}=\left(Z_{2}, Y_{2}, x_{2}\right)$ [22].

\section{Fuzzy Logic Multi-Objective Decision-Making Scheme}

Then, according to the fuzzy set theory, the minimum of the weighted scores is used for the final composite value. The minimum represents the intersection of the sets because all the criteria are necessary to the final decision for load forecasting thermal generation system. For the mathematical equation for load forecasting, the composite index value is represented as follows for the fuzzy set method [28]. The problem of combining typical objectives for multi-objective decision making for load forecasting problem can be considered to involve two aspects resulting in an overall decision function $N$, which is a mapping a decision process, involves the selection of one set of alternative $n_{i}$ into some set $\mathrm{S}$ which has at least a linear ordering on its elements, from a universe of $\mathrm{k}$ alternatives, in particular $N(x)=$ $f\left[n_{1}(x), n_{2}(x), n_{3}(x), \ldots, n_{k}(x)\right]$ for each $x \in X$, where $n_{i}(x)$ indicates the satisfaction of alternative $x$ to objective $n_{i}$ given a set of $d$ objectives or criteria $M=\left\{m_{1}, m_{2}, m_{3}, \ldots, m_{d}\right\}$ that is very important to the multiobjective decision making. For based on the multiobjective decision-making formulation for load forecasting described previously, the decision measure for a particular alternative, $n$, can be replaced with a classical implication of the form.

\section{Electricity Load Forecasting Description using FMODM Method}

For the typical multi-objective decision problem, which a decision problem process, involves the selection of one alternative, $t_{i}$, from a universe of $n$ alternatives.

The use of linguistic variables is currently widespread and the linguistic effect values of load with based on meteorology data alternatives found in this research are primarily used to assess the linguistic ratings given by the evaluators. Furthermore, linguistic variables are used as a way to measure the performance value of load forecasting based on weather meteorology data alternative for each criterion as "Equally", "Low", "Fair", "Good", and "crucial". In Table 1. Explain value membership function of linguistic scale for importance weights of factors for Fuzzy MultiObjective Decision-Making model and Table 2 is 
Table 1. Membership Function of linguistic scale for importance weights of factors

\begin{tabular}{|c|c|}
\hline Definition & Corresponding TFNs \\
\hline No Operation (No) & $(0.0,0.0,0.0)$ \\
\hline Low Important (Li) & $(0.9,0.9,2.9)$ \\
\hline Equally Important (Ei) & $(0.9,2.9,4.9)$ \\
\hline Fair Important (Fi) & $(2.9,4.9,6.9)$ \\
\hline strongly Important $(\mathrm{St})$ & $(4.9,6.9,9)$ \\
\hline Crucial $(\mathrm{Ci})$ & $(6.9,9,9)$ \\
\hline
\end{tabular}

Table 2. Subjective cognition results of evaluators Linguistic variables for the possible rating of success

\begin{tabular}{|c|c|c|c|c|c|}
\hline Evalu & \multicolumn{5}{|c|}{ Linguistic Variables TFNs } \\
\hline & Equally & Low & Fair & Good & Crucial \\
\hline 1 & $\begin{array}{cc}{\left[\begin{array}{ll}0 & 0 \\
40\end{array}\right]}\end{array}$ & $\begin{array}{c}{[40} \\
60 \\
80]\end{array}$ & $\begin{array}{c}70 \\
90 \\
140]\end{array}$ & $\begin{array}{c}{[140} \\
160 \\
180]\end{array}$ & $\begin{array}{c}{[170} \\
200 \\
200]\end{array}$ \\
\hline 2 & $\begin{array}{cc}{\left[\begin{array}{ll}0 & 0 \\
50\end{array}\right]}\end{array}$ & $\begin{array}{c}{[20} \\
60 \\
100]\end{array}$ & $\begin{array}{c}{[60} \\
100 \\
140]\end{array}$ & $\begin{array}{c}130 \\
150 \\
170]\end{array}$ & $\begin{array}{c}{[160} \\
200 \\
200]\end{array}$ \\
\hline 3 & $\begin{array}{cc}{\left[\begin{array}{ll}0 & 0 \\
38\end{array}\right]}\end{array}$ & $\begin{array}{c}{[30} \\
54 \\
86]\end{array}$ & $\begin{array}{c}76 \\
96 \\
130]\end{array}$ & $\begin{array}{c}120 \\
156 \\
180]\end{array}$ & $\begin{array}{c}{[176} \\
200 \\
200]\end{array}$ \\
\hline 4 & $\begin{array}{cc}{\left[\begin{array}{ll}0 & 0 \\
80\end{array}\right]}\end{array}$ & $\begin{array}{c}{[80} \\
100 \\
120]\end{array}$ & $\begin{array}{c}{[120} \\
140 \\
160]\end{array}$ & $\begin{array}{c}{[160} \\
170 \\
180]\end{array}$ & $\begin{array}{c}{[180} \\
200 \\
200]\end{array}$ \\
\hline 5 & $\begin{array}{cc}{\left[\begin{array}{ll}0 & 0 \\
50\end{array}\right]}\end{array}$ & $\begin{array}{c}{[30} \\
60 \\
90]\end{array}$ & $\begin{array}{c}{[90} \\
120 \\
150]\end{array}$ & $\begin{array}{c}150 \\
160 \\
180]\end{array}$ & $\begin{array}{c}{[180} \\
200 \\
200]\end{array}$ \\
\hline
\end{tabular}

Subjective cognition results of evaluators Linguistic variables for the possible rating of success for Fuzzy Multi-Objective Decision-Making model the load prediction unit thermal generation with gas and steam fuel.

\section{Feed Forward Neural Network (FF-NN)}

Artificial Neural Network is a mathematical method inspired by the structure and information processing of biological neural networks. Artificial Neural Networks are intelligent systems that able to learn, memorize and create relationships among data [Chen, at al]. While the standard procedure is to take a rich database that provides the most accurate description of the phenomena or system to be modelled, and then divides it into two sets of data; the training inputs set and the testing or validations set. After the validation or testing test, the Artificial Neural Networks based model will be ready to use. However, for the present application, the Artificial Neural Networks has to start learning based on a weak training set at the beginning, then subsequently develops and sharpens the acquired knowledge while ensuring the continuity of its task.
The period of use of the model delimited by two successive training is the validation period. And the uniqueness of the present modelling approach is that it is progressive, since the artificial neural network model is initially building constructed based on the existing database (of any size), and after this, its training and validations are periodically performed as the database expands over time as explained by Fig. 5.

The Artificial Neural Networks was trained according to the generalized training scheme we can see illustrated by Fig. 6 using the feedforward-NN algorithm. The prediction used mathematic models designed for Fuzzy Multi-Objective Decision Making Feed Forward Neural Network is described in Fig. 7 if the validation test for forecasting the

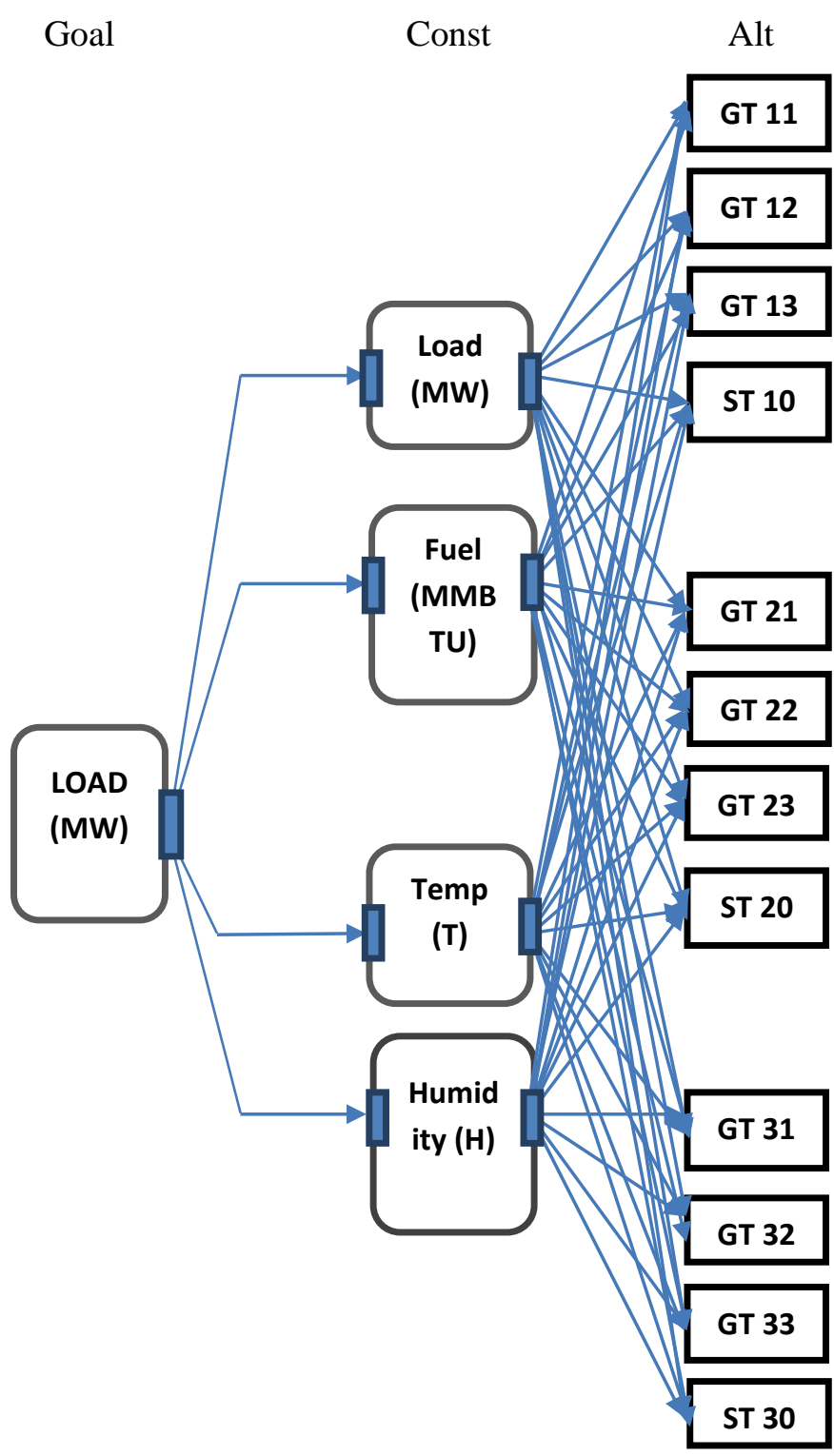

Figure. 4 Process Fuzzy - Multi-Objective Decision Making (FMODM) 


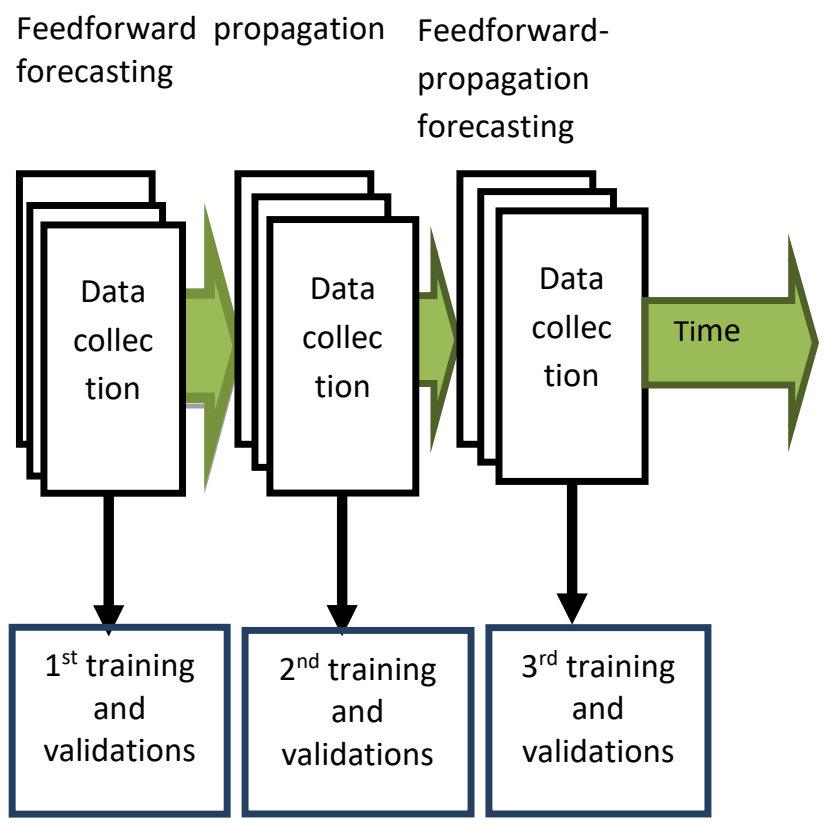

Figure. 5 The progressive Feedforward-Neural Network forecasting

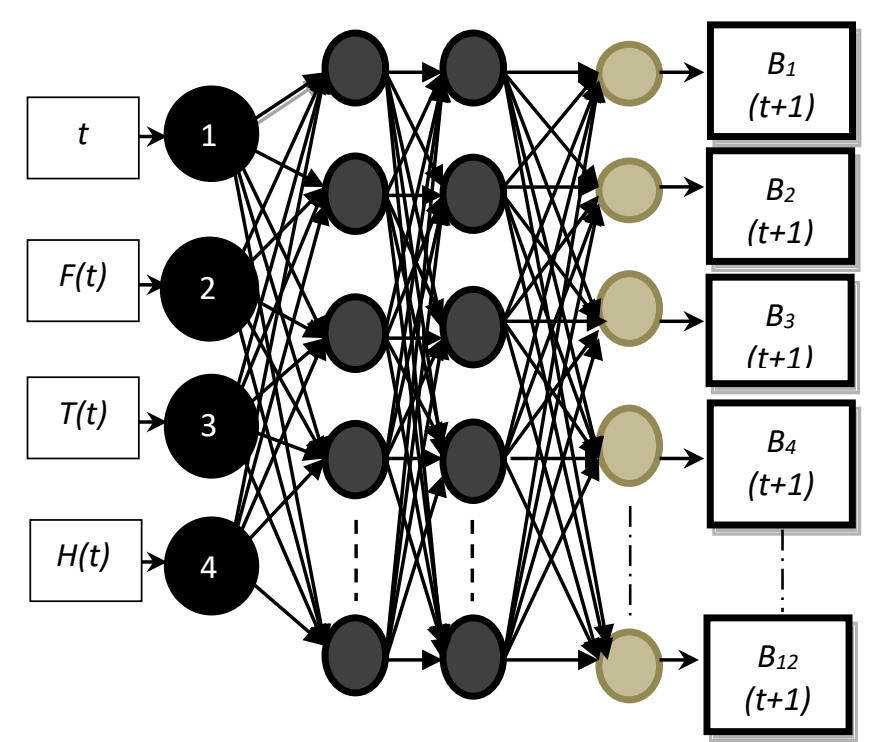

Figure. 6 The Feed Forward structure for forecasting of load

electricity load is successful and gets better the result. The Electricity load prediction process using a hybrid Fuzzy Multi-Objective Decision MakingFeed Forward Neural Network Algorithm can also be divided into two proceesses: (a) Fuzzy MultiObjective Decision-Making mathematics modelling to determine membership function and linguistic variable for input data at the ANN process; (b) The Feedforward Neural Network mathematics for electrical load forecasting. For illustration flowchart, the forecasting process using Fuzzy-Multi Objective Decision Making-Feed Forward Neural Network
Hybrid modelling, consist of: (a) Load for the training set; (b) Load for the testing set; (c) Load for the validation set in Fig. 7. In this research, FuzzyMulti Objective Decision Making-Feed Forward Neural Network structure construction was programmed using MATLAB programming. MATLAB is been provided with some Feedforward Neural Networks tools. In most of the proposed application a typical feed-forward neural network algorithm for load forecasting based on meteorology data in thermal generation structure, shown in Fig. 8, can be expressed by the following and relationship between input data and output of the Fuzzy-Multi Objective Decision Making Feed-Forward Neural Network Hybrid Modelling the forecast Process.

\section{Results and analysis}

This section discusses the result of the FuzzyMulti Objective Decision Making-Feed Forward Neural Network model used to predict future load generation thermal gas by using one hour ahead procedure. The Fuzzy-Multi Objective Decision Making-Feed Forward Neural Network modelling can be divided into three stages: (1) the topology design stage which includes the choice of the FuzzyMulti Objective Decision Making model simulation for the first input using four input variable (2) the second stage Feed-Forward Neural Network model, the number of its layers, the number of neurons in each layer, its inputs and outputs data from FuzzyMulti Objective Decision Making process simulation prediction (3) the training and testing stage during which samples are presented to the feed-forward Neural Network and the weights are adjusted accordingly till a predetermined condition is satisfied; (4) the validation result for the predicted load generation one hour ahead of using FuzzyMulti Objective Decision Making Feed-Forward Neural Network model. The procedure is described in Fig. 9. We test our model using previously described databases for load generation forecasting based on target data parameters meteorology data, during the process forecast of 1 hour ahead.

\subsection{Input data}

Input data for the Fuzzy-Multi Objective Decision Making Feed-Forward Neural Network model, it is expected that a valid result of load generation based on meteorological predict will be produced. The design model forecast is divided into two stages:

Step 1: Calculating weight using the membership function based on the Fuzzy Multi Objectif Decision 
Making model every hour for all of the thermal gas generation.

Step 2: Using input data for Feed Forward Neural Network modeling and simulation based on the assumption that the existing data input testing and training is a combination of the results obtained from the data Fuzzy Multi-Objective Decision Making model.

The Fuzzy-Multi Objective Decision Making Feed-Forward Neural Network model proposed in this research seeks to estimate and forecast a thermal gas station production for one hour. Fig. 9 illustrates an example of normalized data for sixteen hours from the user database and the Fuzzy MultiObjective Decision Making Feed Forward hybrid model every hour for sixteen-hour for all of the gas and steam thermal load generation. In Fig. 10. The result describes the comparison of load generation forecasting in sixteen-hours (00:30 to 16:00) on March 20, 2018, based on the Fuzzy-Multi Objective Decision Making Feed-Forward Neural Network model, actual data, k-Nearest Neighbour Neural Network model, and Artificial Neural Network model. The value result shows that a very Short-term load generation prediction simulation using the Fuzzy Multi-Objective Decision Making Feed Forward Neural Network model during sixteen hours window gives better compared to the simulation k-Nearest Neighbour-Neural Network model and Artificial Neural Network model. From the simulation results using the F- Multi-Objective Decision Making Feed Forward Neural Network simulation model based on meteorological data consists of load generation for gas and steam thermal generation, cost, fuel, temperature, and humidity. Mean Square Error (MSE) and Root Mean Square Error (RMSE) was used as an error statistical indicator of the forecast load generation hybrid modelling. Prediction from the Fuzzy-Multi Objective Decision-Making model was then compared with the Feed Forward Neural Network model and the mean average of meteorological data prediction. For the comparison, the Fuzzy MultiObjective Decision Making Feed Forward NN hybrid model performed only 1000 iterations for each learning period. The Feed Forward Neural Network model is initially constructed based on the Fuzzy Multi-Objective Decision-Making model based on meteorology data, and after that, its testing and training input data using a hybrid model based on meteorology data is periodically performed as the database expands over time. The hybrid FuzzyMulti Objective Decision Making Feed-Forward Neural Network model is good agreement with
Table 3. The combinations for the Feed Forward Neural Networks topology

\begin{tabular}{|l|c|}
\hline Number of hidden layers & 1 \\
\hline Number of hidden neurons & 16 \\
\hline $\begin{array}{l}\text { Activation function used in the hidden } \\
\text { layer }\end{array}$ & tansig \\
\hline $\begin{array}{l}\text { Activation function used in the output } \\
\text { layer }\end{array}$ & Purelin \\
\hline Target Error & 0.001 \\
\hline Learning Rate & 0.1 \\
\hline
\end{tabular}

measured data in the object position gas and steams thermal generation. To analyse and evaluate the performance of the models, a statistical error measurement was used in the experiment, namely the mean square error and root mean square error. And in Table 3. explain about the combination for fuzzy multi-objective decision making feed-forward neural network proposed hybrid model.

For the comparison, using the Fuzzy MultiObjective Decision Making Feed Forward Neural Network hybrid model performed a maximum of 1000 epoch for each learning period. In Fig. 7 illustrates the all the data for the three subsets: (a) load for the training data set; (b) load for the testing data set; and (c) load for the validation data set. In this figure the plots illustrations for load versus the time. The feed-forward Neural Networks algorithm is initially constructed for the training based on the Fuzzy Multi-Objective Decision-Making model data, and after this, its training is periodical as the database expands over time.

To analysis, the accuracy of each model to forecast the load generation value, mean square error, and root mean square error coefficient between value results of Fuzzy-Multi Objective Decision Making Feed-Forward Neural Network and actual ground measurements were calculated. The result very short term for 1 hour ahead load generation steam and gas thermal forecasting we can see Fig. 11. In Fig. 12. (a) and (b) Illustration about calculation with mean square error and root mean square error coefficient between actual data FuzzyMulti Objective Decision Making Feed-Forward Neural Network hybrid model, k-Nearest Neighbour-Neural Network model and Artificial Neural Network model.

For more clearly, the lowest error statistical indicators of k-Nearest Neighbour-Neural Network modelling mean square error with a value 0.01 and root mean square error with a value 0.22 during the sixteen periods while the highest root means square error with a value 6.0 MW and the highest mean square error with a value $1366.82 \mathrm{MW}$, and Artificial Neural Network modelling mean square 
error with a value 0.01 and root mean square error with a value 0.24 during the sixteen periods while the highest root means square error with a value 6.05 MW and the highest mean square error with a value $1367 \mathrm{MW}$. On the other hand, the error statistical indicator for the proposed hybrid model (Fuzzy Multi-Objective Decision MakingFeedForward Neural Network model) root mean square error is value error 0.2 and mean square error is value error 0.001 .

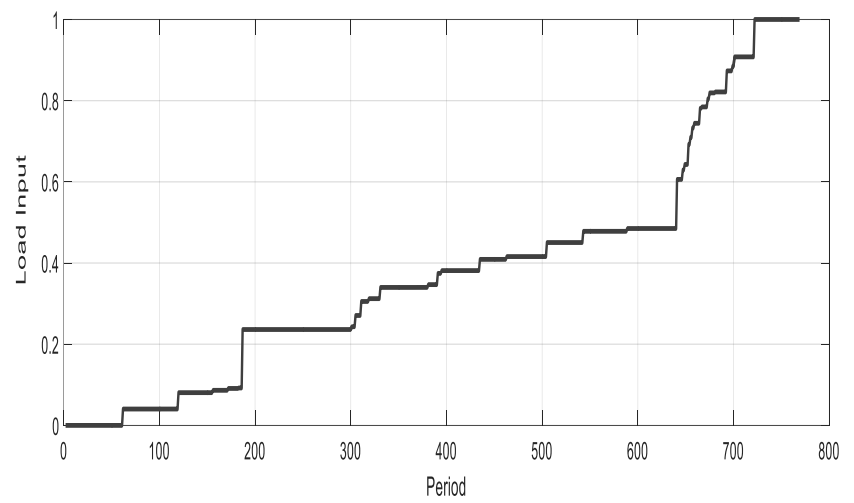

(a)

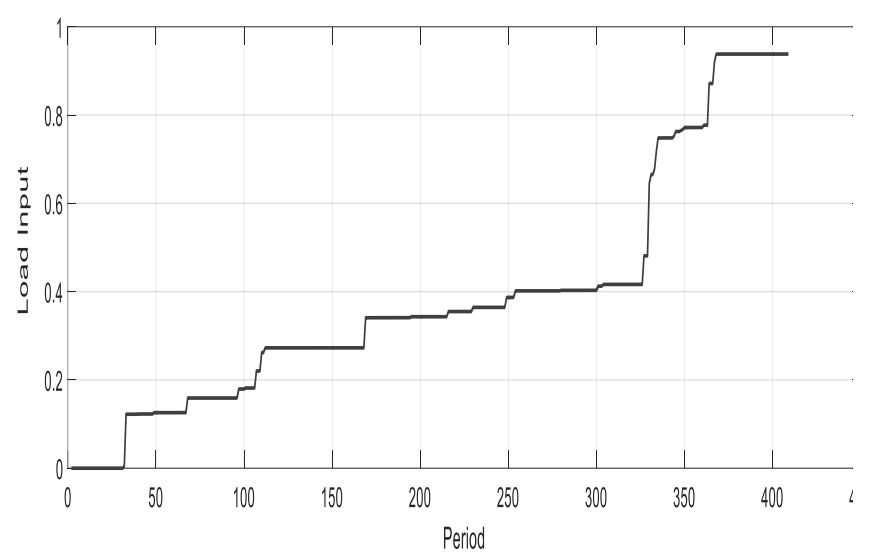

(b)

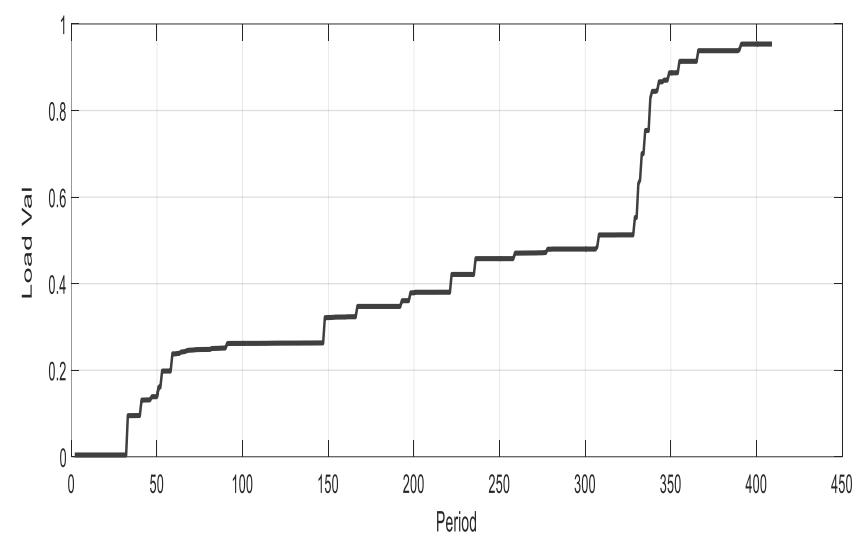

(c)

Figure 7. (a) Load for the training set, (b) Load for the testing set, and (c) Load for the validation set

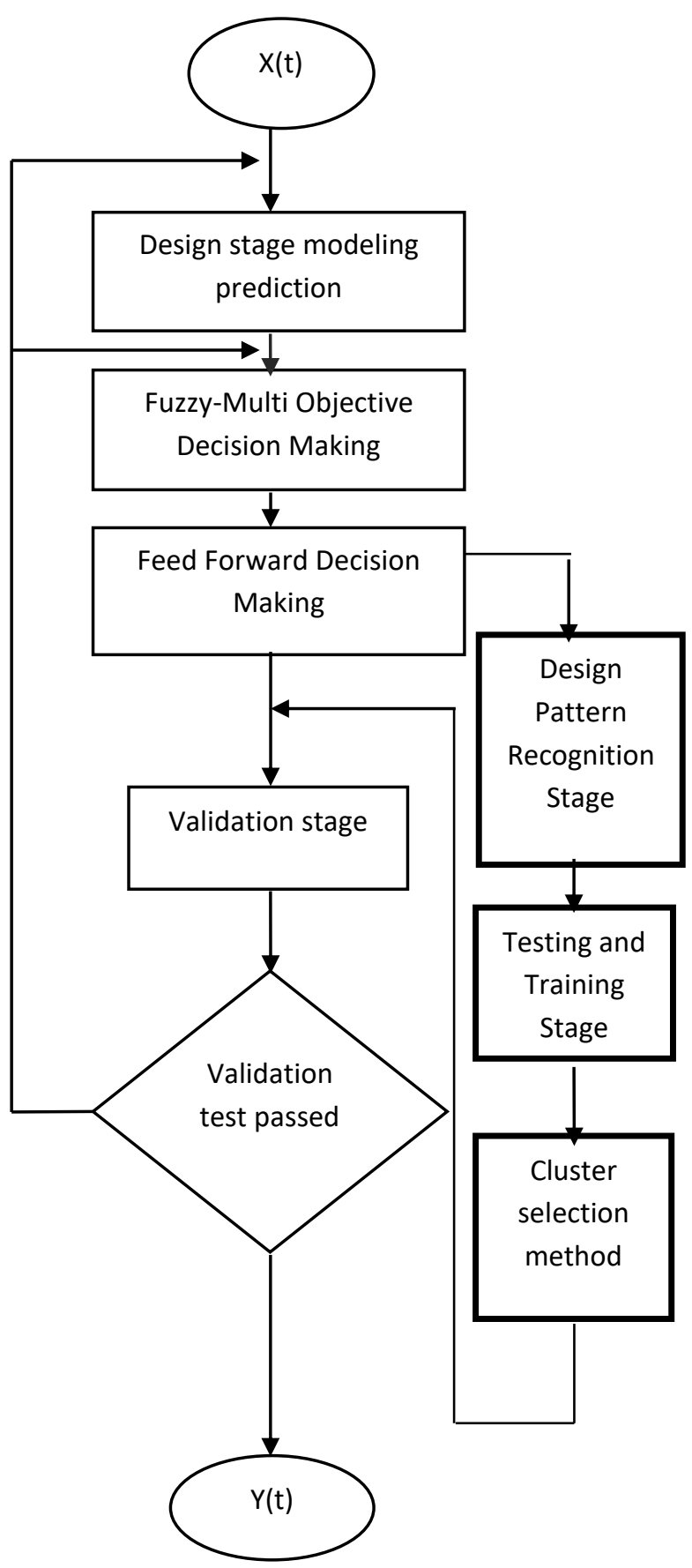

Figure. 8 The flowchart of the Fuzzy-Multi Objective Decision Making Feed-Forward Neural Network Hybrid Modelling the forecast Process

Note that the highest Root Mean Square Error valued 4,43 MW and Mean Square Error was 387.8 MW during the sixteen periods.

The Fuzzy Multi-Objective Decision MakingFeedforward Neural Network hybrid model displays a better forecast than the k-Nearest Neighbor Neural Network model and Artificial Neural Network analysis simulation load generation. 


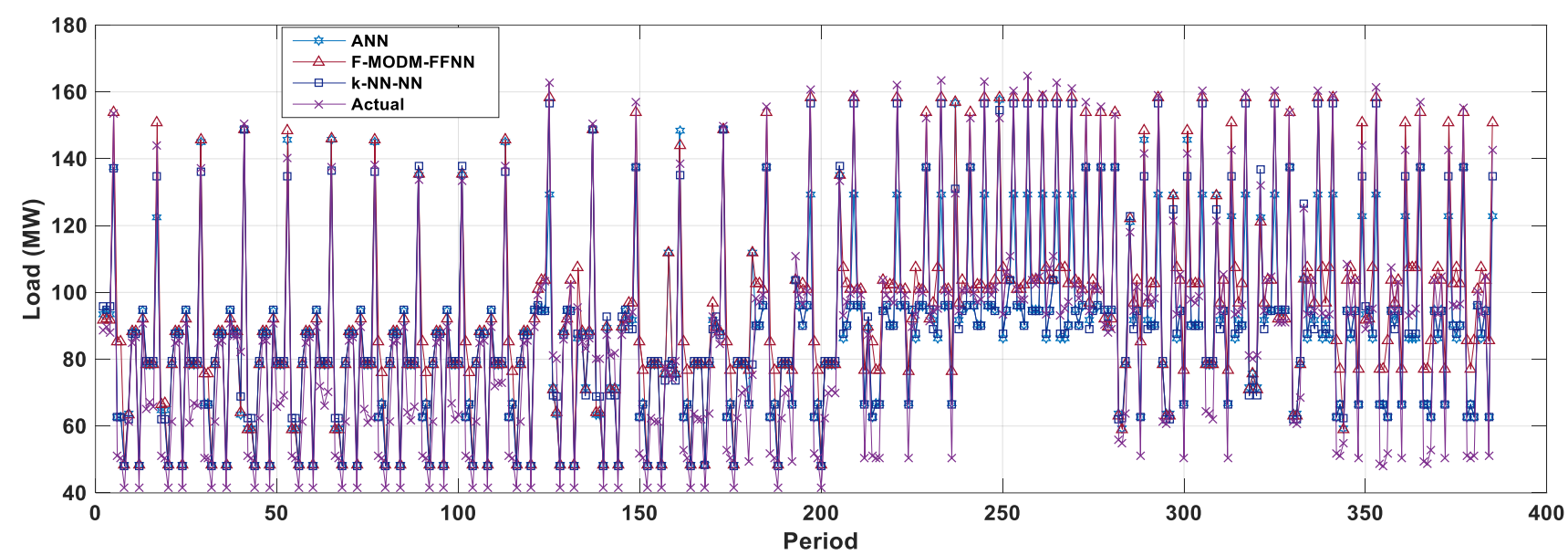

Figure. 10 Load generation thermal gas based on the Fuzzy Multi-Objective Decision Making Feed Forward Neural Network (F-MODM-FFNN) hybrid modelling (from 00:30 a.m. to 04:00 p.m.)

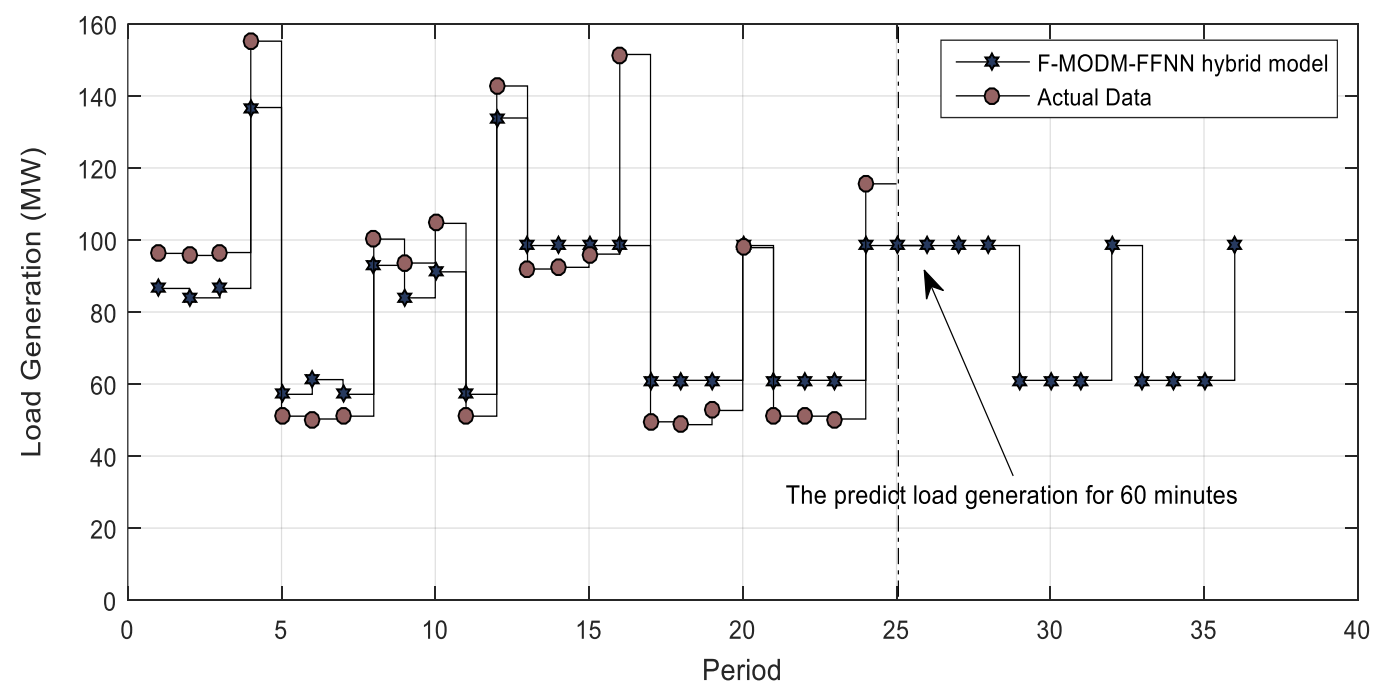

Figure. 11 The result very short term for 1 hour ahead load predict gas and steam generation using hybrid model versus actual data

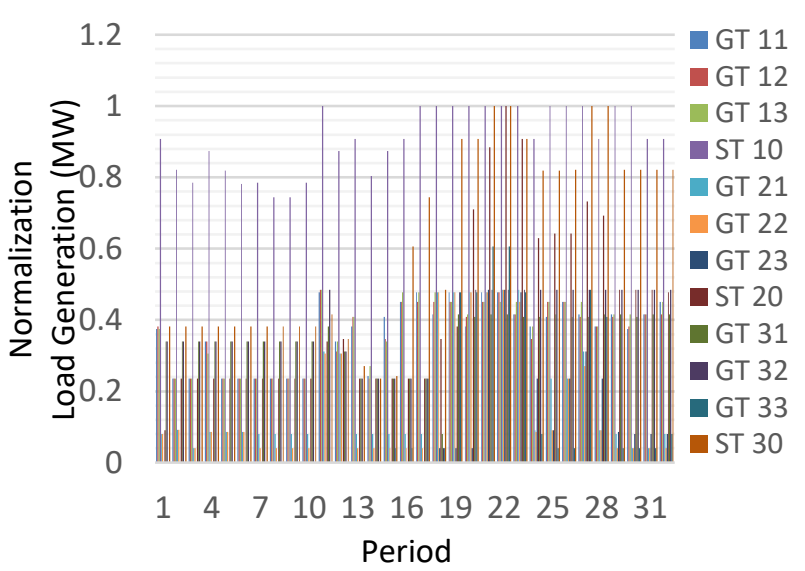

Figure. 9 Normalization input data Fuzzy Multi-Objective Decision Making Feed Forward Neural Network (FMODM-FFNN) simulation and modelling (from 00:30 a.m. to 04:00 p.m)

\section{Conclusion}

In this work, a novel methodology Fuzzy MultiObjective Decision Making-Feed Forward Neural Networks modelling for very short-term forecasting specifically 60 minutes or 1 hour ahead of load generation has been developed. In this research, we proposed a new hybrid model for load generation forecasting using a combination of Fuzzy-Multi Objective Decision Making modelling and Feed Forward Neural Network. The proposed model in research accepts as input parameters the mean hourly load thermal and gas generation, the sixteen hours air temperature, the sixteen hours fuel every thermal and gas generation, and the sixteen hours humidity. The output is represented by the 1 hour or 60 minutes ahead of load generation. The following conclusions can be drawn from this study: 


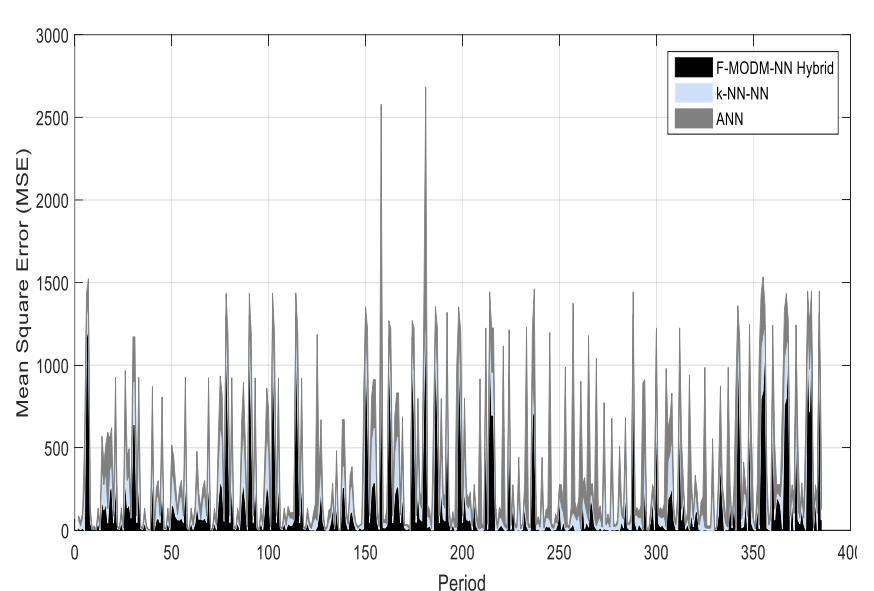

(a)

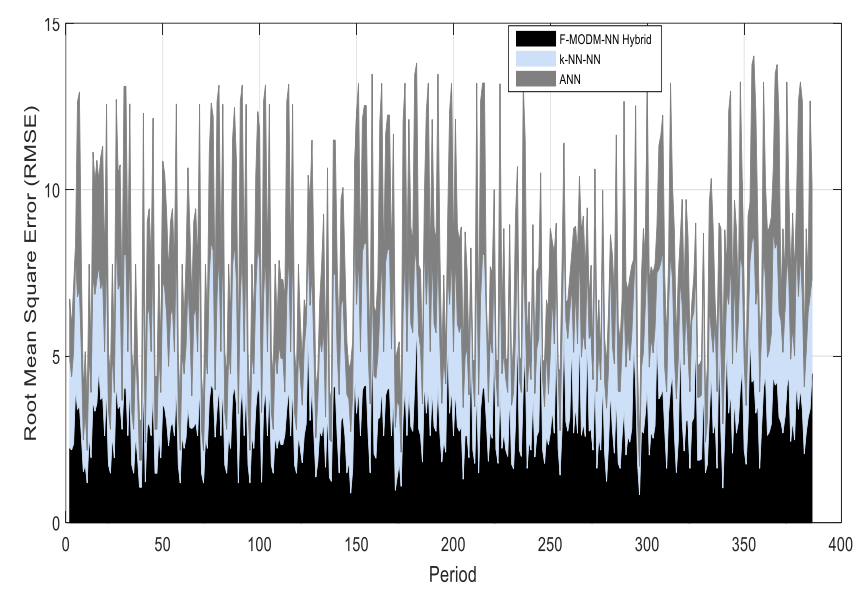

(b)

Figure. 12 (a) Mean Square Error (MSE) coefficient between load generation Predict based on Fuzzy-Multi Objective Decision Making Feed-Forward Neural

Network hybrid model, k-Nearest Neighbour-Neural

Network and Artificial Neural Network model and (b)

Root Mean Square Error (RMSE) coefficient between load generation Predict based on Fuzzy-Multi Objective Decision Making Feed-Forward Neural Network hybrid model, k-Nearest Neighbour-Neural Network and Artificial Neural Network model

- A proper selection of features derived from the load time series is necessary to maximize the fuzzy multi-objective decision-making feedforward neural network forecast performance.

- The result of fuzzy multi-objective decision making feed-forward neural network hybrid model used for 1 hour ahead short term load forecast for Gresik in Indonesia, shows that hybrid model has a good performance and reasonable prediction accuracy was achieved for this model. The result suggests that fuzzy multi-objective decision making feed-forward neural network hybrid model with the developed structure and topology can perform good prediction with least error and finally this fuzzy multi-objective decision-making feedforward neural network could be an important tool for short term load forecasting. This research concerns how to predict load generation data at thermal generation. The study also considers the availability of a local measured database. It clearly shows that the load generation forecasting using a different $\mathrm{F}$ MODM-FFNN hybrid model for every hour based on meteorological data giving a better result output, which means the load generation forecasting largely depends on variable meteorological data, where the meteorology data variables consist of load, humidity, and temperatures.

A comparison between the actual data measures of the load generation Fuzzy Multi-Objective Decision Making-Feed Forward Neural Network model has better and predictor shows a good forecast performance for 1 hour ahead and compared with the k-Nearest Neighbor-Neural Network model and Artificial Neural Network model. The root means square error statistical indicators of the k-Nearest Neighbour-Neural Network model is the root mean square error value 0.22 and mean square error is mean value 0.01 and compared with Artificial Neural Network model with the value the root mean square error statistical indicators of the Artificial Neural Network model is mean error value 0.24 and mean square error is mean value 0.01 . On the other hand, the error statistical indicator for the proposed model FuzzyMulti Objective Decision Making-Feed Forward Neural Network with a value the mean square error is mean value error 0.001 and root mean square error is mean value error 0.2. Note that the highest root means square error was $4.43 \mathrm{MW}$ and meansquare error was $387.8 \mathrm{MW}$ during the sixteen periods. The performance of the proposed FuzzyMulti Objective Decision Making-Feed Forward Neural Network hybrid model predicts shows good predicted performance compared with only the kNearest Neighbour-Neural Network model and Artificial Neural Network. And the proposed Fuzzy Multi-Objective Decision Making-Feed Forward Neural Networks hybrid model in this research can be used effectively to forecast very short term the load thermal and gas generation by giving the resulting output closer and match with actual measured data. The further scope of this paper is towards the forecasting development of a new load generation interconnection system hydro thermal modelling through which major obstacles like Occlusions and Background Movements with 
using calculate minimalization transmission losses and position generation. Furthermore, a new frame selection technique which can able to long term load generation predicted linear derive both linear and non-linear relationships between action sequences is intended to develop.

\section{Conflicts of Interest}

The authors declare no conflict of interest.

\section{Author Contributions}

Eppy Yundra and Unit Three Kartini conceptualization and designed model; Eppy Yundra and Laili Ika Wardani performed the experiments; Eppy Yundra, Unit Three Kartini, Dwi Ardianto and Laili Ika Wardani contributed investigation, software, materials, validation, reagents, data curation and formal analysis tools; Eppy Yundra and Unit Three Kartini writing original draft preparation, review and editing, Unit Three Kartini and Eppy Yundra, project administration, and funding acquisition.

\section{Acknowledgments}

Authors gratefully and wish to thank acknowledge the support Department of Electrical Engineering and Intelligence Power System Lab. for their valuable suggestions as well as Universitas Negeri Surabaya.

\section{References}

[1] D. Liu, L. Zeng, C. Li, K. Ma, Y. Chen, and Y. Cao, "A Distributed Short-Term Load Forecasting Method Based on Local Weather Information", IEEE Systems Journal, Vol. 12, No. 1, pp. 1937-9234, 2016.

[2] Y. Wang, Q. Chen, M. Sun, C. Kang, and Q. Xia, "An Ensemble Forecasting Method for the Aggregated Load with Sub Profiles", IEEE, Vol. 9, No. 4, pp. 1949-3063, 2018.

[3] T. Vincent, P. Audrey, G. Yannig, A. Anestis, and P. Jean-Michel, "Electricity Forecasting Using Multi-Stage Estimator on Nonlinear Additive Models", IEEE Transactions on Power Systems, Vol. 31, No. 5, pp. 3665-3673, Sept 2016.

[4] M. Liu, Y. Shi, and F. Fang, "Load Forecasting and Operation Strategy Design for CCHP Systems Using Forecasted Loads", IEEE Transactions on Control Systems Technology, Vol. 23, No. 5, pp. 1063-6536, 2015.
[5] T.Hong, J. Wilson, and J. Xie, "Long Term Probabilistic Load Forecasting and Normalization With Hourly Information", IEEE Transactions on Smart Grid, Vol. 5, No. 1, pp. 456-462, 2014.

[6] B. Liu, J. Nowotarski, T. Hong, and R. Weron, "Probabilistic Load Forecasting via Quantile Regression Averaging on Sister Forecasts", IEEE Transactions on SmartGrid, Vol. 8, No. 2 pp. 730-737, 2017.

[7] B. Li, J. Zhang, Y. He, and Y. Wang, "Short Term Load Forecasting Method Based On Wavelet Decomposition With Second-Order Gray Neural Network Model Combined With ADF Test", IEEE Access, Vol. 5, pp. 1632416331, 2017.

[8] K. Weicong, D. Z. Yang, D. J. Hill, L. Fengji, and Y. Xu, "Short-Term Residential Load Forecasting Based on Resident Behaviour Learning", IEEE Transactions on Power Systems, Vol. 33, pp. 1087-1088, 2018.

[9] J. Xie and T. Hong, "Temperature Scenario Generation For Probabilistic Load Forecasting", IEEE Transactions on Smart Grid, Vol.9, pp. 1680-1687, 2018.

[10]L. Franklin, L. W. Jen, H. Heng, Y. D. Wang, and L. S. Robert, "Using Smart Meter Data to Improve the Accuracy of Intraday Load Forecasting Considering Customer Behavior Similarities", IEEE Transactions on Smart Grid, Vol. 6, pp. 911-918, 2014.

[11]S. Cai, L. Liu, H. Sun, and J. Yan, "Fisher Information Based Meteorological Factors Introduction and Features Selection for Short Term Load Forecasting”, Entropy, Vol. 20, No. 184, pp. 2-17, 2018.

[12]B. Wang, Y. Li, and J. Watada, "Supply Reliability and Generation Cost Analysis Dual Load Forecast Uncertainty in Unit Commitment Problems", IEEE Transactions on Power Systems, Vol. 28, pp. 1-10, 2013.

[13] U. T. Kartini, D. Ardianto, and W. Laili, "Very Short-Term Load Forecasting Based On Meteorological With Modeling k-NN-Feed Forward Neural Network", J. Electrical Systems, Vol. 15, pp. 1-16, 2019.

[14]F. Mohamad, J. The and H. Abunima, "MultiObjective Optimization of Solar/Wind Penetration in Power Generation Systems", IEEE Access, Vol. 7, pp. 169094-169106, 2019.

[15]D. J. Y. Tey, Y. F. Gan, G. Selvachandran, S. G. Quek, F. Smarandache, L. H Son, M. A. Basset, and H. V Long, "A Novel Neutrosophic Data Analytic Hierarchy Process for Multi-Criteria Decision-Making Method: A Case Study in 
Kuala Lumpur Stock Exchange", Special Section on New Trends In Brain Signal Processing and Analysis, IEEE Access, Vol. 7, pp. 53687-53697 2019.

[16] S. M. A. Pahnehkolaei, H. Vahedi, A. Alfi, and K. Al-Haddad, "Comparative Study of MultiObjective Finite Set Predictive Control Methods With New Max-Min Strategy Applied On A Seven-level Packed U-Cell Inverter", IET Power Electrons, Vol. 12, pp. 2170-2178, 2019.

[17]E. Barocio, J. Regalado, E. Cuevas, F. Uribe, P. Zuniga, and P. J. R Torres, "Modified BioInspired Optimisation Algorithm With a Centroid Decision Making Approach For Solving A Multi-Objective Optimal Power Flow Problem", IET Generation, Transmission \& Distribution, Vol. 11, pp. 1012-1022, 2016.

[18]Z. Deng, Z. Lu, Z. Guo, W. Yao, W. Zhao, B. Zhao, and C. Hong, "Coordinated Optimization of Generation and Compensation to Enhance Short Term Voltage Security of Power Systems Using Accelerated Multi-Objective Reinforcement Learning”, IEEE Access, Vol. 8, pp. 34770-34782, 2020.

[19] H. Farzin, M. F. Firuzabad, and M. M Aghtaie, "A Stochastic Multi-Objective Framework For Optimal Scheduling of Energy Storage Systems in Microgrids", IEEE Transactions on Smart Grid, Vol. 8, pp. 117-127, 2017.

[20] M. S. Javadi, M. Saniei, H. R. Mashhadi, and G. G Alcaraz, "Multi-objective Expansion Planning Approach: Distant Wind Farms and limited Energy Resources Integration", IET Renewable Power Generation, Vol. 7, pp. 652668, 2013.

[21] H. Khazraj, B. Y Khanghah, P. Ghimire, F. Martin, M. Ghomi, F. F. Silva, and C. L. Bak, "Optimal Operational Scheduling and Reconfiguration Coordination in Smart Grids For Extreme Weather Condition", IET Generation, Transmission \& Distribution, Vol. 13, pp. 3455-3463, 2019.

[22] H. Mavalizadeh, A. Ahmadi, and A. Heidarr, "Probabilistic Multi-objective Generation and Transmission Expansion Planning Problem Using Normal Boundary Intersection", IET Generation, Transmission \& Distribution, Vol. 9, pp. 560-570, 2015.

[23] S. M. Mazhari, H. Monsef, and R. Romero, "A Multi-Objective Distribution System Expansion Planning Incorporating Customer Choices on Reliability", IEEE Transactions on Power Systems, Vol. 31, pp. 1330-1340, 2016.

[24] S. Motepe, A. N. Hasan, and R. Stopforth, "Improving Load Forecasting Process For a
Power Distribution Network Using Hybrid AI and Deep Learning Algorithms", IEEE Access, Vol. 7, pp. 82584-82598, 2019.

[25] M. Zhang and Y. Li, "Multi-Objective Optimal Reactive Power Dispatch of Power Systems by Combining Classification Based MultiObjective Evolutionary Algorithm and Integrated Decision Making”, IEEE Access, Special Section on Artificial Intelligence Technologies for Electric Power Systems, Vol. 8, pp. 38198-38209, 2020.

[26] A. Ouammi, "Optimal Power Scheduling for a Cooperative Network of Smart Residential Buildings", IEEE Transactions on Sustainable Energy, Vol. 7, pp. 1317-1326, 2015.

[27] T. Ouyang, Y. Ho, H. Li, Z. Sun, and S. Back, "Modeling and Forecasting Short-Term Power Load With Copula Model and Deep Belief Network", IEEE Transactions on Emerging Topics In Computational Intelligence, Vol. 3, No. 2, pp. 127-136, 2019.

[28] Y. Shen and G. Ge, "Multi-Objective Particle Swarm Optimization Based on Fuzzy Optimally", IEEE Access, Vol. 7, pp. 101513101526, 2019.

[29] A. Shukla, K. Verma, and R. Kumar, "Multiobjective Synergistic Planning of EV FastCharging Stations In The Distribution System Coupled With The Transportation Network", IET Generation, Transmission \& Distribution, Vol. 13, No. 15, pp. 3421-3432, 2019.

[30]A. Trivedi, D. Srinivisan, D. Sharma, and C. Singh, "Evolutionary Multi-Objective DahAhead Thermal Generation Scheduling in Uncertain Environment", IEEE Transactions on Power Systems, Vol. 28, No. 2, pp. 1345-1354, 2013.

[31]Z. Zhao, J. Yang, W. Yang, H. Luo, and Y. Peng, "Coordinated Multi-Objective Optimization for Multiple Trade-Offs in Pumped Outage Condition of Pumped Storage System", IEEE Access, Vol. 7, pp. 161394161411, 2019. 